\title{
CORONARY ANGIOGRAPHY AFTER ROSC: IS ECG WITHOUT STEMI STILL AN OPEN QUESTION?
}

\author{
Scapol S1, Rakar S1, Vitrella G1, Fabris E1, Perkan A1, Cageggi2 D, Zambon \\ M2, Peratoner A2, Roman Pognuz E3, Antonaglia V2, Berlot G3, Sinagra G1
}

\section{Department of Cardiology1 , Emergency Medical System2,}

Institute of Anesthesiology and Intensive Care3

\section{BACKGROUND}

Despite the improvement on the field and in the intensive care unit, the survival rate of OHCA remains poor. The main cause of OHCA is myocardial ischemia. If the first ECG after ROSC is STEMI the guidelines recommend the early CA, what about no-STEMI it's still an open question.

\section{METHODS}

We retrospectively analyzed data from our registry, Trieste Cardiac Arrest Registry, which collects the cases of OHCA of presumed cardiac origin. We compare two population of no-STEMI, who underwent to CA and who did not. All patients with shockable rhythm and STEMI ECG after ROSC immediately underwent to CA. No-STEMI patients underwent to CA within 2 hours if clear ischemic causes were present or previously subjected to a fast rule out of extra cardiac etiology at ER.

\section{RESULTS}

From 2011 to 2015 in Trieste we collected 544 cases of OHCA, first rhythm was shockable in $29.7 \%$,ROSC was present in $33.9 \%$. STEMI patients was $38 \%$ and no-STEMI 62\%. All STEMI patients underwent CA, in the $27.5 \%$ CAD was $>50 \%$ and $100 \%$ in $67.5 \%, 68 \%$ were treated with PCI.

\begin{tabular}{ll}
\hline \multicolumn{2}{c}{ POPULATION } \\
\hline & $544 \mathrm{pts}(\%)$ \\
VF/VT & 29.7 \\
ROSC & 33.9 \\
STEMI & 38 \\
NO-STEMI & 62 \\
\hline
\end{tabular}

\begin{tabular}{ll}
\hline \multicolumn{2}{c}{ STEMI 62 PTS } \\
\hline & 38 pts (\%) \\
CA & 100 \\
CAD $>50 \%$ & 27.5 \\
CAD 100\% & 67.5 \\
PCI & 68 \\
\hline
\end{tabular}

No-STEMI patients, $52.5 \%$ had ischemic myocardial disease, myocardial dysfunction was present in $36.8 \%, 74 \%$ did echocardiography,32.1\% did CT to exclude other causes. The 55\% underwent CA,CAD was $<50 \%$ in $16.3 \%, \geq 50 \%$ in $27.9 \%$ and occlusive in $34.9 \%$. We treated with PCI the $11.3 \%$ and TTM the $42.1 \%$ of no-STEMI patients.

Among no-STEMI patients we compared who underwent CA and who did not. Who did CA had higher prevalence of shockable rhythm (61 vs $25, \mathrm{p} 0.001)$ and a better chance of discharge(53 vs 30 , p0.040), survival rate at 30days(61.4 vs 36, p0.025)at 1year(38.6 vs 16.7, p0.031)and better neurological outcome(47 vs 36, p0.296).

\begin{tabular}{lc}
\hline \multicolumn{2}{c}{ NO STEMI 62 PTS } \\
\hline & $\%$ \\
CPT ischemic & 52.5 \\
$\mathrm{EF}<55$ & 36.8 \\
TEE HR & 74 \\
$\mathrm{CT}$ & 32.1 \\
$\mathrm{CA}$ & 55 \\
$\mathrm{CAD}<50$ & 16.3 \\
$\mathrm{CAD} \geq 50$ & 27.9 \\
$\mathrm{CAD} 100$ & 34.9 \\
$\mathrm{PCI}$ & 11.3 \\
$\mathrm{TTM}$ & 42.1 \\
\hline
\end{tabular}

CORONARY ARTERY DISEASE

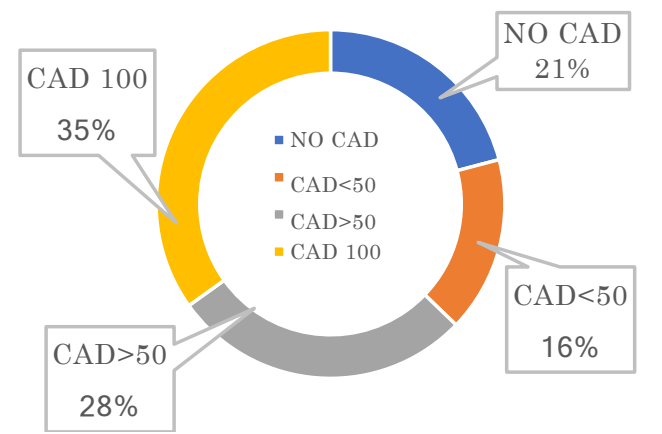

\section{CONCLUSION}

The study of no-STEMI patients with CA had strong impact on their survival, due to PCI or focusing on other therapies. All patients who survived OHCA of suspected cardiac origin, should be referred to a center with the availability of CA, regardless of post-ROSC ECG. 